\title{
Laboratory tests of two-dimensional wavelet trigger in radio detection of cosmic rays
}

\author{
Zbigniew Szadkowski \\ University of Łódź \\ Department of Physics and Applied Informatics \\ ul. Pomorska 149, 90-236 Łódź, Poland \\ Email: zszadkow@kfd2.phys.uni.lodz.pl
}

\author{
Anna Szadkowska \\ Łódź University of Technology \\ Center of Mathematics and Physics \\ ul. Żeromskiego 116, 90-024 Łódź, Poland \\ Email: aszadkow@p.lodz.pl
}

\begin{abstract}
The origin of ultrahigh-energy cosmic rays (UHE$\mathrm{CRs})\left(\mathrm{E} \geq 10^{17} \mathrm{eV}\right)$ is a fundamental question of astroparticle physics. The induced shower of secondary particles in the atmosphere of the Earth provides essential information on the cosmic ray itself: arrival direction, primary energy, and mass. In the air shower many electrons and positrons form a pancakeshaped particle front with a typical thickness less than $1 \mathrm{~m}$ close to the shower axis to more than $10 \mathrm{~m}$ far from the shower axis The geomagnetic field induces a drift velocity in these particles which is perpendicular to the direction of the initial cosmic ray. The generated current is a source of coherent emission of electromagnetic waves at wavelengths larger than the size of the dimension of the charge cloud i.e., for radio frequencies in the range of 30-300 $\mathrm{MHz}$.

The radio technique allows a detail study of the electromagnetic part of an air shower in the atmosphere and provide information complementary to that obtained by surface detectors water Cherenkov tanks, which are predominantly sensitive to the muonic content of an air shower at the ground. One of the promising attempts to observe UHECRs by the detection of their coherent radio emission is a wavelet trigger based on a FPGA.

The paper presents first laboratory results from the twodimensional wavelet trigger, implemented into the prototype Front-End Board developed for the Auger surface detector based on the Cyclone ${ }^{\circledR} V$ FPGA 5CEFA9F31I7. The wavelet trigger investigates a distribution of partial power contributions for two Fourier indices, simultaneously in time and frequency domains. Preliminary results are very promising and show that the wavelet trigger could improve a radio detection system
\end{abstract}

\section{INTRODUCTION}

$\mathbf{T}$ HE Pierre Auger Observatory is the ground based detector of the ultrahigh-energy cosmic rays (UHECR) [1] It contains from three types of detectors: fluorescence (FD), surface (SD) and radio (RD). The present challenges of the RD are to understand the emission mechanisms and the features of the radio signal, and to develop an adequate measuring instrument. Electron-positron pairs generated in the shower development are separated and deflected by the Earth magnetic field, hence introducing an electromagnetic emission [2]-[4]. During shower development, charged particles are concentrated in a shower disk a few meters thick. This results in a coherent radio emission up to about $100 \mathrm{MHz}$. Short but coherent radio pulses of duration from $10 \mathrm{~ns}$ up to several $100 \mathrm{~ns}$ are generated with an electric field strength increasing approximately linearly with the energy of the primary cosmic particle inducing the extended air showers (EAS), i.e. quadratic dependence of the radio pulse energy vs. primary particle energy [5]-[9]. In contrast to the fluorescence technique with a duty cycle of about $10 \%$ (fluorescence detectors can operate only during moonless nights [10]), the radio technique allows nearly fulltime measurements and long-range observations because of the high transparency of the air to radio signals in the investigated frequency range.

One of the currently developing techniques is radio signal power estimation based on wavelet transforms, which can be an alternative proposal to the algorithms currently in operation.

The radio signal is spread over time intervals in the order of 200 nanoseconds; most registered samples have a time interval below $150 \mathrm{~ns}$. The frequency window for the maximal antenna efficiency is $\sim 30-80 \mathrm{MHz}$ [11]. This range is additionally filtered by a band-pass filter. According to Nyquist's theorem the sampling frequency should be twice as high as the maximal frequency in an investigated spectrum. The anti-aliasing filter should have the cut-off frequency of $\sim 85 \mathrm{MHz}$. Taking into account the width of the transition range for the filter (from pass-band to stop-band) the final sampling frequency should not be lower than $170 \mathrm{MHz}(200 \mathrm{MHz}$ in our test setup and measurements).

\section{WAVELETS}

The contribution of non-stationary power at many different frequencies [12] in ADC traces (a time series X, with values of $x_{n}$, at time index $\mathrm{n}$ ) is analyzed by the wavelet transform. Each ADC sample is separated in time by a constant time interval $\Delta \mathrm{t}$. The wavelet transform $W_{n}(s)$ is a convolution of the wavelet function $\psi$ with our original time series:

$$
W_{n}(s)=\sum_{m=0}^{N-1} x_{m} \psi^{*}\left[\frac{(m-n) \Delta t}{s}\right]
$$

where $\mathrm{s}$ denotes the frequency scale and the asterisk (*) complex conjugate, $\mathrm{N}$ - number of ADC samples involved in a wavelet approximation, respectively.

A convolution in time domain corresponds to a simple product of Fourier coefficients in frequency domain. Fourier coefficients for reference wavelets are pre-calculated and are implemented into the FPGA code as constants. We need only to calculate on-line N-point Fourier coefficients of ADC 


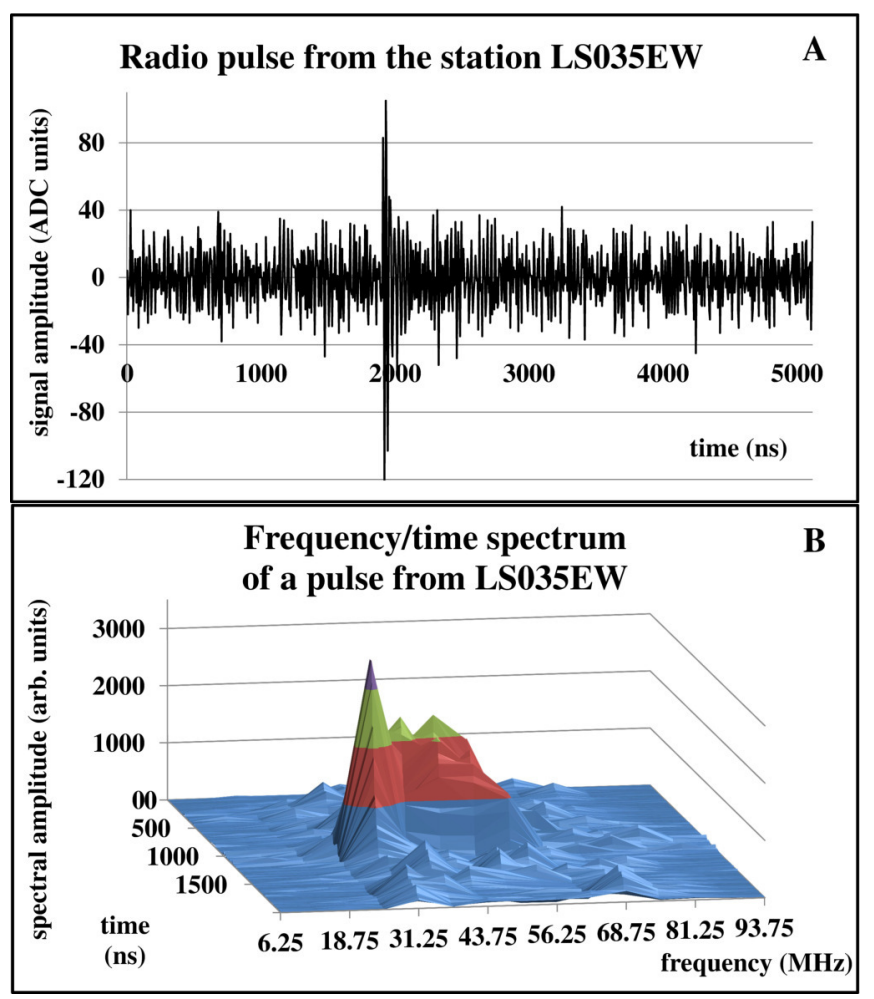

Fig. 1. Real AERA event for the radio station LS035EW

samples Selected $\mathrm{N}=32$. The FPGA routine of 32-point FFT (Fig. 1 in [13]) shows a sufficient speed for the FPGA 5CEFA9F31I7, the heart of the new Front-End Board for the prototype Auger surface detector, which contains 3 inputs for 3 PMTs from the water Cherenkov surface detector +2 inputs for the radio detector with two polarizations.

In order to get a 2-dim frequency/time distribution of a temporary signal power, the convolution (1) should be cal-

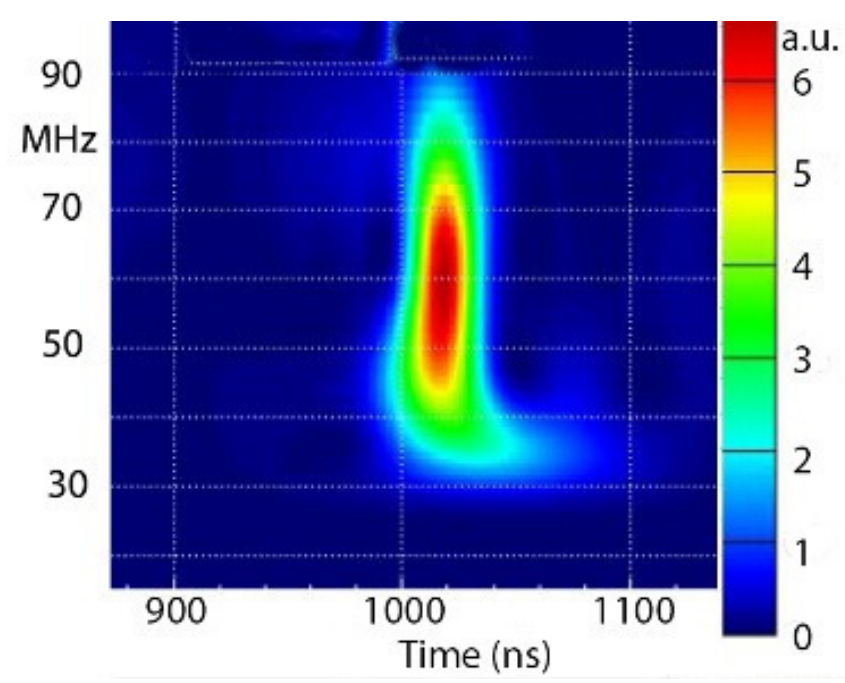

Fig. 2. High-resolution distribution of a power of a real cosmic ray event
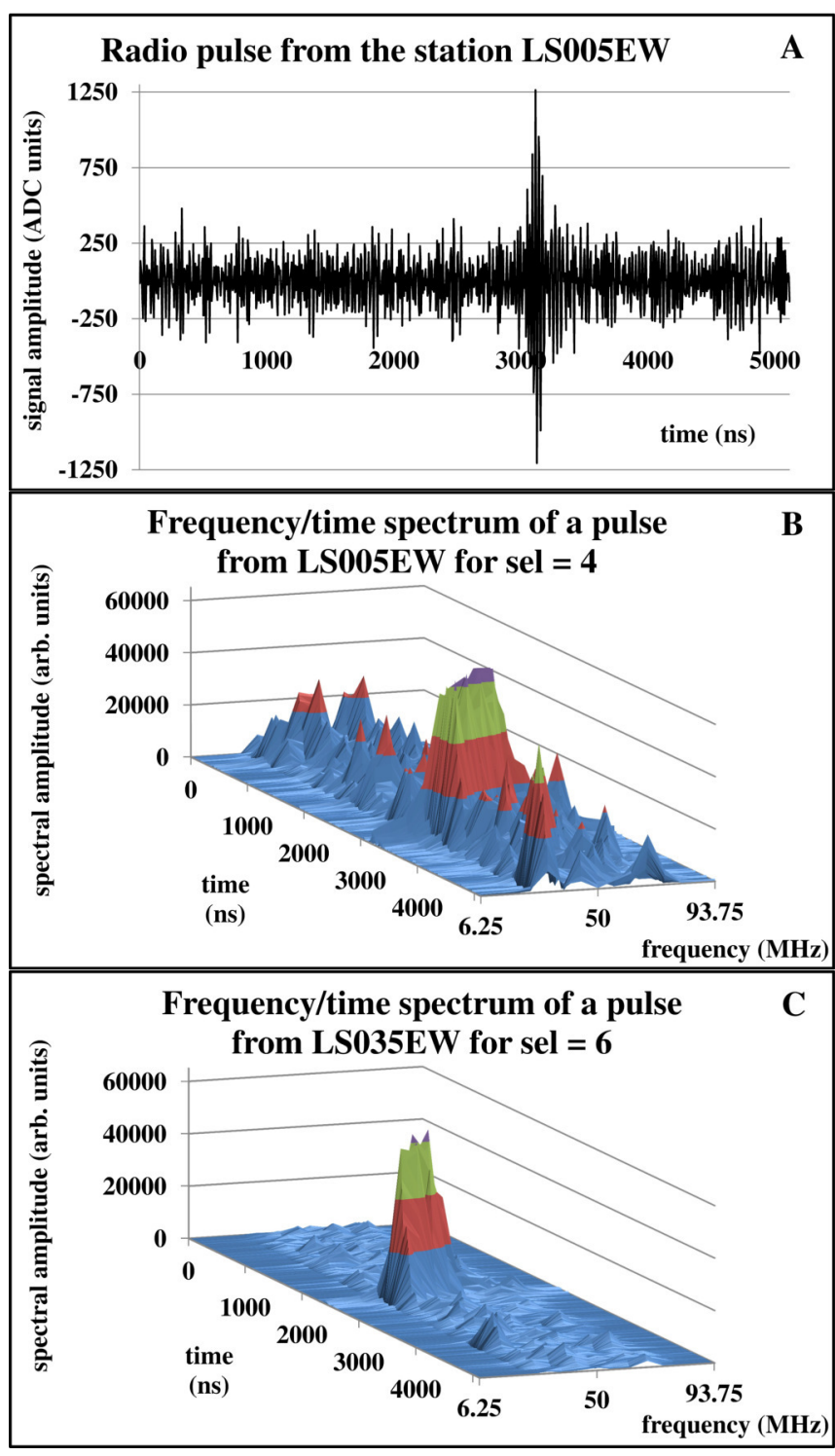

Fig. 3. Real AERA event for radio station LS005EW

culated $\mathrm{M}$ times for each scale s. M is a compromise between a frequency resolution and a resources utilization, which determines a registered performance of the FPGA implementation. The global wavelet power spectrum is defined as $\left|W_{n}(s)\right|^{2}[14]$ and the total signal power $\bar{W}^{2}$ can be expressed as follows:

$$
\sum_{j=0}^{M-1}\left|W_{j}\right|^{2}=\frac{1}{N} \sum_{j=0}^{M-1} \sum_{k=0}^{N-1}\left|\bar{X}_{k} \times \bar{\Psi}_{k, j}\right|^{2}
$$

The 32-point FFT routine gives only 16 independent FFT coefficients due to well known their symmetry. For $200 \mathrm{MHz}$ sampling, the grid is $6.25 \mathrm{MHz}$. Only 15 wavelet engines were implemented for 15 FFT real and imaginary coefficients from 6.25 to $93.75 \mathrm{MHz}$.

Several families of reference Morlet wavelets were tested for the scaling factor $s^{-1}=\alpha$, e.g. $0.04,0.01$ and 0.004 . 


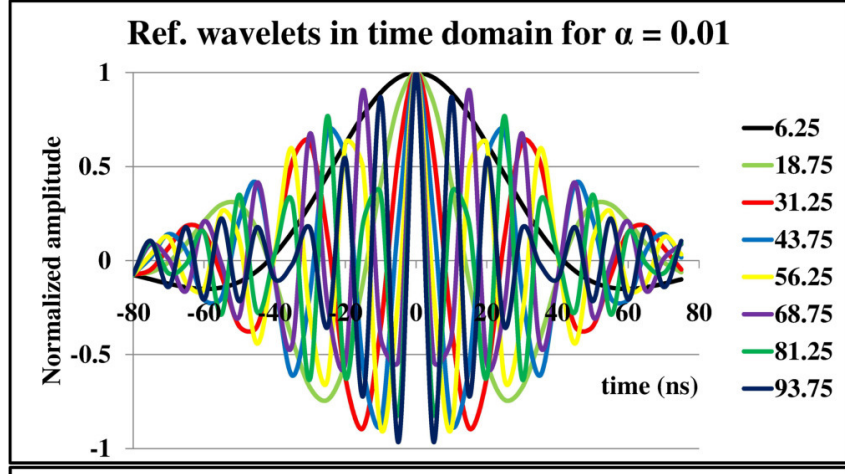

Ref. wavelets in time domain : $\alpha=0.001$

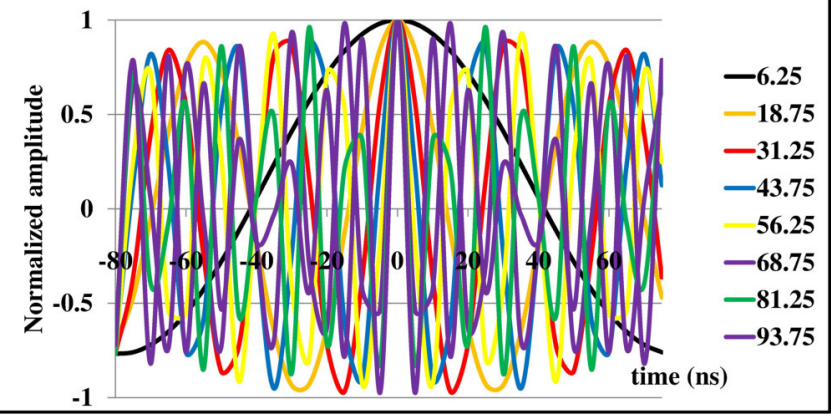

Fig. 4. The Morlet reference wavelets for 6.25-93.75 MHz investigating range for $\alpha=0.01$ and $\alpha=0.001$, respectively. Wavelets for $\alpha=0.01$ cover almost exactly the investigating time window. For $\alpha=0.001$ wavelets are far outside the time window. Practically, the time window is analyzed in an uniform way.

$$
f_{\alpha, \text { freq }}(k)=\cos \left(2 \pi k \frac{\text { freq }}{\text { sampling }}\right) \exp \left(-\alpha \cdot k^{2}\right)
$$

where $-16 \leq k \leq 15$, sampling $=200(\mathrm{MHz})$.

Wavelets for $\alpha=0.04$ are squeezed to non-negligible values in a range of $-10 \leq k \leq 10$. For $\alpha=0.004$, wavelets are stretched to a larger range beyond $-16 \leq k \leq 15$. The factor $\alpha=0.01$ covers almost perfectly the time window of 32-points (Fig. 4A). The factor $\alpha=0.001$ covers the time window almost in an uniform way (Fig. 4B). I.e timing of signals does not correlate with reference wavelets, as e.g. for $\alpha=0.01$.

Fig. 5 shows a contribution to the power spectrum from neighboring wavelets due to a spectral leakage. For $\alpha=$ 0.001 the spectral leakage is almost negligible. Practically, the system will work on a base of pure Fourier coefficients. For the $\alpha=0.01$ at least two neighboring frequency bins (giving a significant contribution) have to be taken into considerations. This requires a usage of several multipliers. However, for $\alpha=$ 0.01 the spectral amplitudes of neighboring frequency bins are roughly twice lower, corresponding values are simply cropped by removing the LSB according to:

$$
W_{n}[13 . .0]=\bar{X}_{n}[13 . .0]+\bar{X}_{n-1}[13 . .1]+\bar{X}_{n+1}[13 . .1]
$$

where $W_{n}$ the power for the n-wavelet, $\bar{X}_{n}, \bar{X}_{n-1}$ and $\bar{X}_{n+1}$ modules of Fourier coefficients (corresponding to the contribution of power from wavelets: main with the index $n$

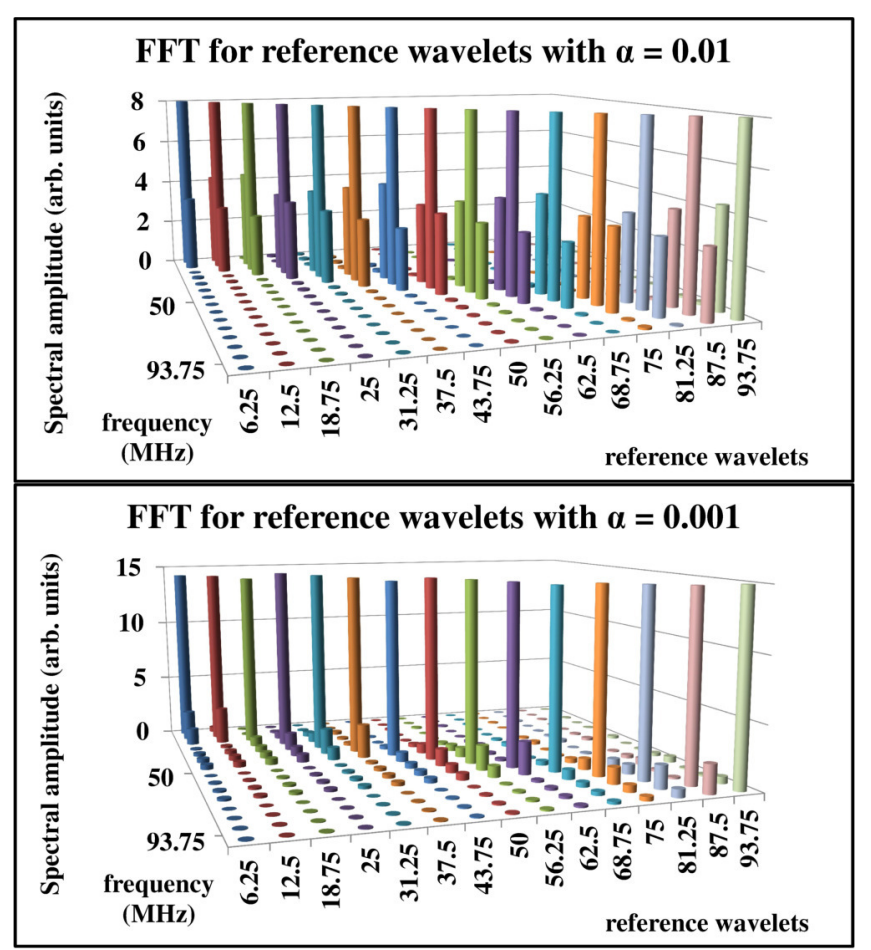

Fig. 5. The Fourier spectrum for 15 reference wavelets scaled for $\alpha=0.01$ and $\alpha=0.001$, respectively.

and neighboring with indices $n-1$ and $n+1$.). Multipliers can be replaced by the sum of the peak with two additional neighboring frequency bins. The FPGA sum routine is shown in Fig. 4. Outputs from 15 sum routines (wavelet engines) are inputs to the 2-dimensional array in Fig. 7.

\section{FPGA IMPLEMENTATION}

Modules:

$$
\left\|W_{n}\right\|=\bar{X}_{n, R e}^{2}+\bar{X}_{n, I m}^{2}
$$

are calculated by the Altera ${ }^{\circledR}$ IP routine ALTMULT_ADD. Altera ${ }^{\circledR}$ provides also a routine allowing a calculation of a square root ALTSQRT, however, the routine significantly slows down the global registered performance even for a large latency (additional pipeline stages for a speed improvement). We had to operate with $\left\|W_{n}\right\|$ modules to keep $200 \mathrm{MHz}$ global clock.

The algorithm of the wavelet trigger has been published in [13]. It has been implemented into Altera ${ }^{\circledR}$ Cyclone ${ }^{\circledR} \mathrm{V}$ E FPGA 5CEFA9F31I7, the heart of the new prototype FEB developed for the upgraded surface detector in AugerPrime [15][19]. After the conversion from the LVDS ADC outputs to a single-ended internal standard of the FPGA fabric signals are put on the wavelet engine.

15 outputs corresponding to 15 frequencies from 6.25 to 93.75 MHz with a $6.25 \mathrm{MHz}$ grid are pipelined in 15 chains of shift registers (Fig. 7). Outputs from this matrix drive comparators with fixed threshold set by the NIOS ${ }^{\circledR}$ processor $15 \times 32$ matrix of comparator outputs are next Ex-NORed with 


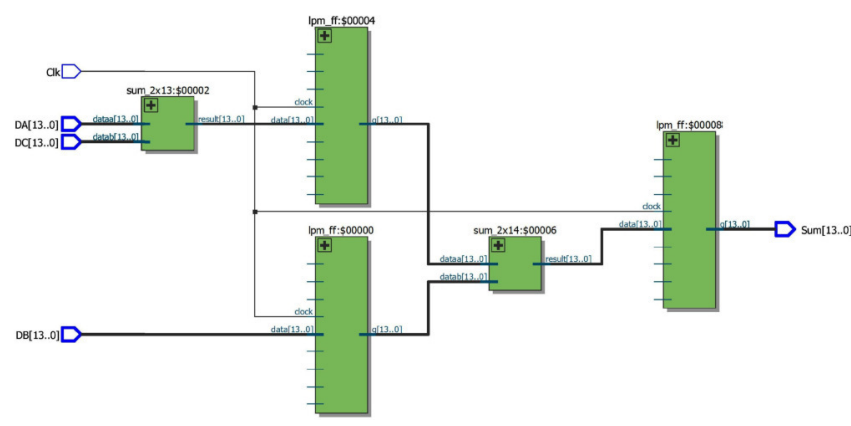

Fig. 6. The FPGA routine calculating wavelet contributions from neighboring frequency bins.

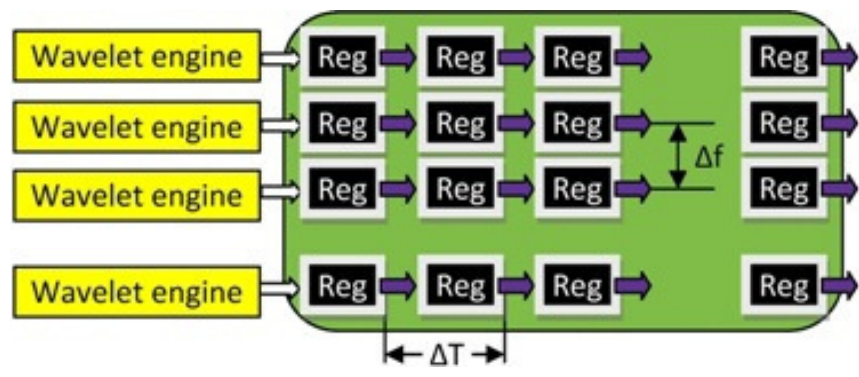

Fig. 7. The scheme of the 2-dimensional analysis. Partial power estimators calculated for reference wavelets spread in the frequency domain with the grid $\Delta \mathrm{f}$ are propagated in the registered pipelines to be available simultaneously for series time bins shifted by $\Delta \mathrm{T}$

the $15 \times 32$ matrix of patterns (Fig. 10) established by the NIOS $^{\circledR}$ processor. Positive recognized Ex-NOR conditions are summed and give 10-bit "occupancy" power estimator. A trigger is generated if the online calculated "occupancy" is above an arbitrary threshold, again set by the NIOS ${ }^{\circledR}$ processor. The same algorithm has been also implemented into the Cyclone ${ }^{\circledR}$ IV EP4CE75F29C6 FPGA. This FPGA is currently in use in the real radio stations. The resource occupancy of the wavelet engine for EP4CE75F29C6 is on the same level as for 5CEFA9F31I7, both for the "pure" wavelet engine and the trigger with the NIOS. For a full configuration the resource occupancy is relatively high, however, the register performance is still above $200 \mathrm{MHz}$ with a sufficient safety margin which allows an operation of the algorithm simultaneously with the standard code. The FPGA used in Dutch radio stations is

TABLE I

RESOURCES OCCUPATION OF WAVELET ENGINE FOR THE FPGA CURRENTLY IN USE : CYCLONE IV E EP4CE75F29C6 AND THE FPGA IN THE PROTOTYPE FRONT-END BOARD : 5CEFA9F31I7 FROM CYCLONE V E FAMILY

\begin{tabular}{|c||c|c|}
\hline FPFA & EP4CE75F29C6 & 5CEFA9F31I7 \\
\hline logic elements & $21737 / 75408(29 \%)$ & \\
\hline ALMs & & $11488 / 113560(10 \%)$ \\
\hline Total registers & 16195 & 20499 \\
\hline 9-bit multipliers & $162 / 400(42 \%)$ & $54 / 342(16 \%)$ \\
\hline DSP blocks & & $2541 \mathrm{k} / 12220 \mathrm{k}(21 \%)$ \\
\hline Memory bits & $2038 \mathrm{k} / 2745 \mathrm{k}(74 \%)$ & \\
\hline
\end{tabular}
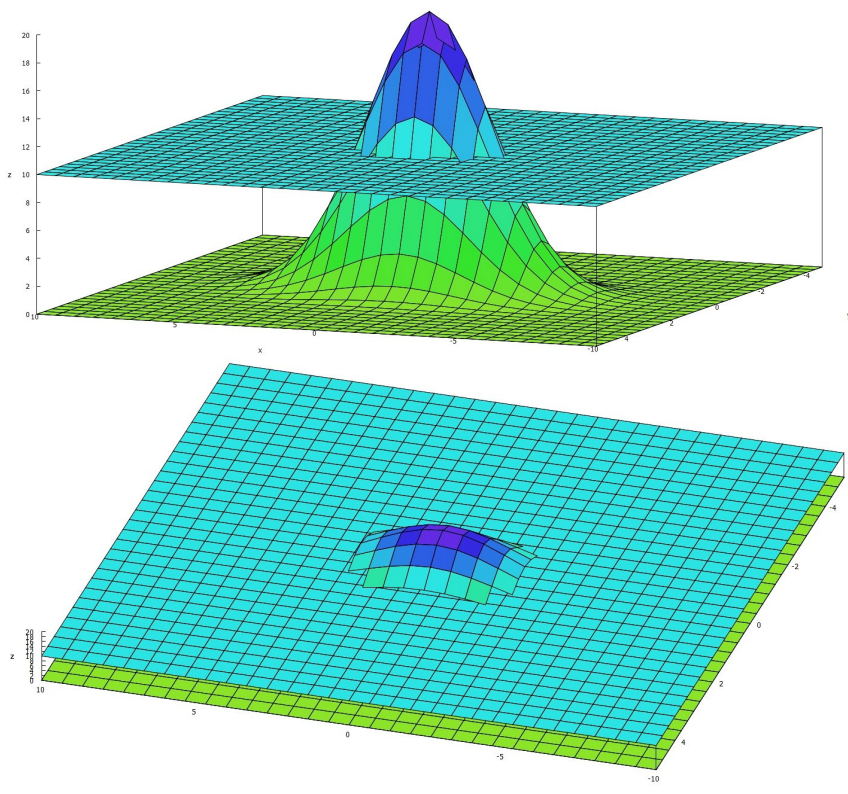

Fig. 8. Illustration of a calculation of power over the threshold. Here, the threshold $=10$ (arb. units) while signal power corresponds to the twodimensional "Gauss bell". The upper graph shows two-dimensional distribution of signal power with a surface cutting the "bell" on the threshold level The lower graph shows the part above the threshold interesting for the trigger.

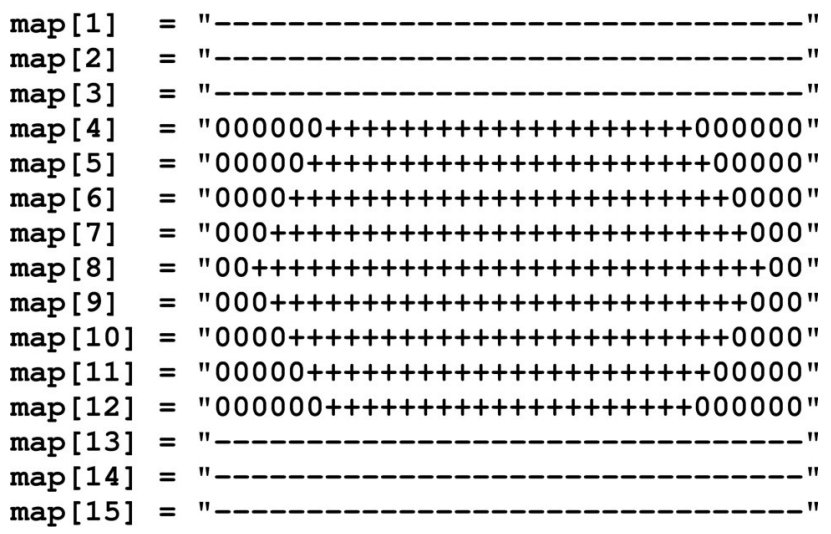

Fig. 10. An example of expected pattern. map[i] correspond to frequencies from 6.25 to $93.75 \mathrm{MHz}$ range $(\mathrm{i}=1, \ldots, 15)$ with $6.25 \mathrm{MHz}$ grid. The accepted frequency range for a signal detection is within $25-75 \mathrm{MHz}$ (map[4] $\operatorname{map}[12])$.

EP4C75F29C6 from Cyclone ${ }^{\circledR}$ IV E Altera family. This chip is large enough the wavelet trigger to be implemented and tested in real environment of Argentinean pampas.

\section{TWO DIMENSIONAL TRIGGER}

Wavelet power coefficients are propagated in the set of shift registers creating a two-dimensional matrix with a power distribution (Fig. 7). A set of comparators detect the active areas of power vs time and frequencies to estimate a probability of an existence of real signal. Fig. 8 shows a principle of a detection of an example signal. The two-dimensional set of registers (Fig. 7) creates a two-dimensional distribution of 

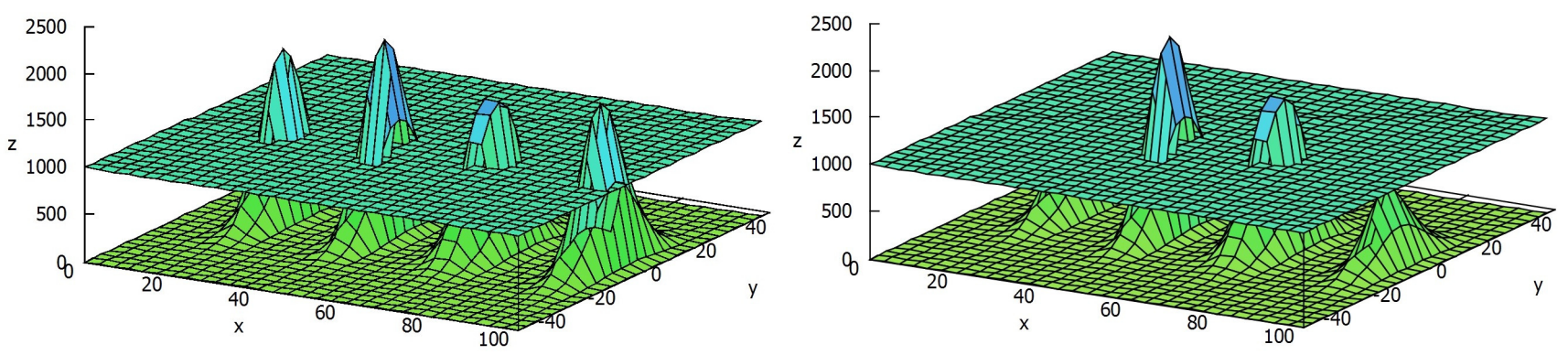

Fig. 9. 3D-plots generated by MAXIMA for arbitrarily assumed frequency spectrum of artificially generated pulse by iFFT with 4 peaks above the threshold (corresponding to $17.12,40.9,65.2$ and $90.7 \mathrm{MHz}$, respectively, left graph) and for with only 2 peaks (corresponding to 40.9 and $65.2 \mathrm{MHz}$, respectively, right graph). The axis $x$ corresponds to the frequency range from 0 to $100 \mathrm{MHz}$, the axis $y$ denotes time evolution, the axis $z$ is in arbitrarily units corresponding to (6).

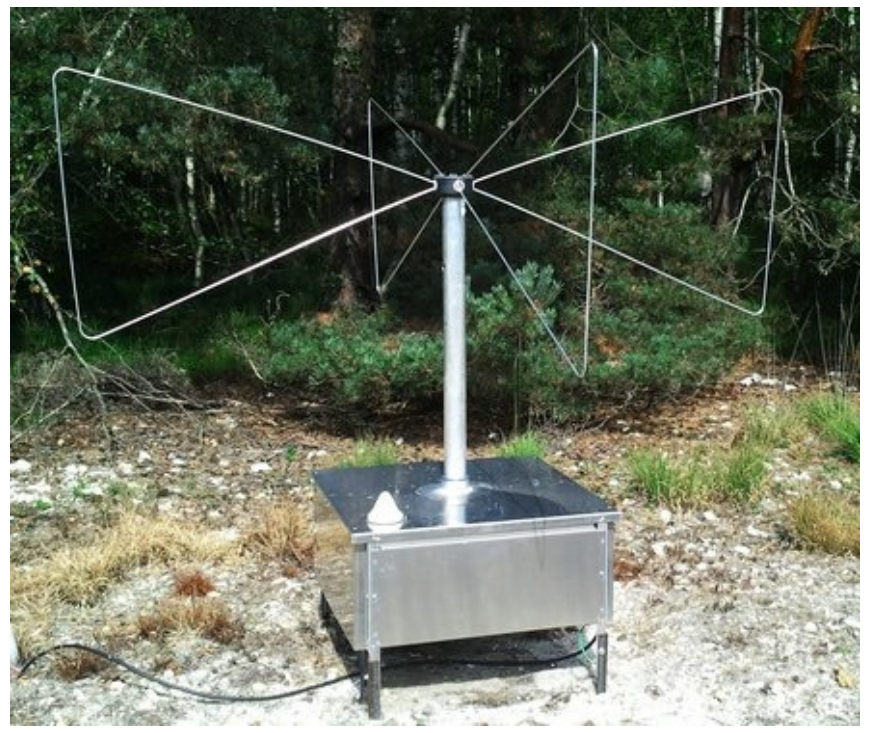

Fig. 11. The Pierre Auger radio station [18]

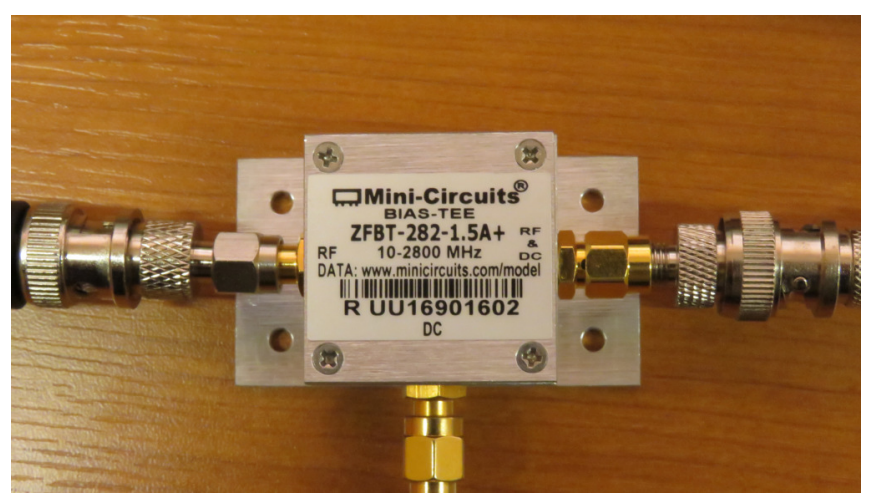

Fig. 12. Circuit which allows driving analog ASIC via antenna cable.

signal power simultaneously for time and frequency. The flat surface in Fig. 8 corresponds to a threshold, which cuts-off a part of distribution lying above the surface. These parts create flat distributions of active power regions.

The power distributions are next compared with an expected

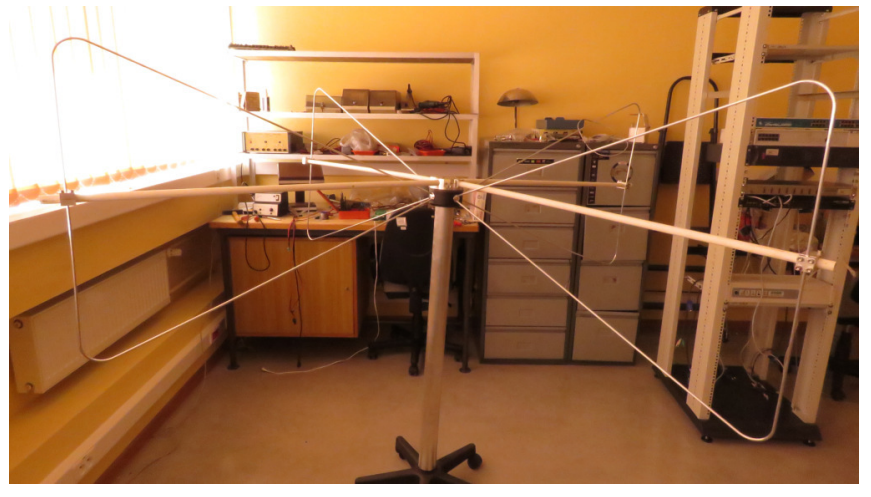

Fig. 13. AERA antenna in the Łódź laboratory for test of various RFI filters and wavelet trigger

patterns generated by the NIOS ${ }^{\circledR}$ processor, which transfers the pattern to the FPGA fabric to some dedicated registers. Comparator results are next Ex-NORed with the expected pattern (an example shown in Fig. 10). Ex-NORed 1-bit signals are next summed to get an "occupancy" in a power space. If the "occupancy" is greater than an arbitrary value (also established by the NIOS $^{\circledR}$ processor), the final trigger is generated. Internal frequencies (marked as "+") give a positive contribution, but peripheral ones (marked as "--") give negative contribution. Zeros limit the time range to 28 time bins.

\section{AdVANCED TESTS}

For tests, a pulse (Fig. 17A) with the following frequency spectrum (Fig. 9 - simulated by MAXIMA and Fig. 18B measured in the FEB) was generated

$$
f(t)=\sum_{k=0}^{3} A_{j} * \exp ^{-\frac{\left(\left(t-f_{k}\right)^{2}\right)}{\left(2 * \sigma_{k}^{2}\right)}}
$$

where amplitudes: $A_{0}=1700, A_{1}=2213, A_{2}=1500, A_{3}=$ 1800 (in ADC units), frequencies: $f_{0}=17.12, f_{1}=40.9, f_{2}$ $=65.2, f_{3}=90.7$ (in $\mathrm{MHz}$ ) and variances: $\sigma_{0}^{2}=862, \sigma_{1}^{2}=$ 662, $\sigma_{2}^{2}=1262, \sigma_{3}^{2}=762$, respectively (Fig. 9).

According to our expectation the spectrum contains two peaks for very low $(\sim 18.25 \mathrm{MHz})$ and very high $(\sim 93.75$ $\mathrm{MHz}$ ) frequencies, which are out of range for a standard 

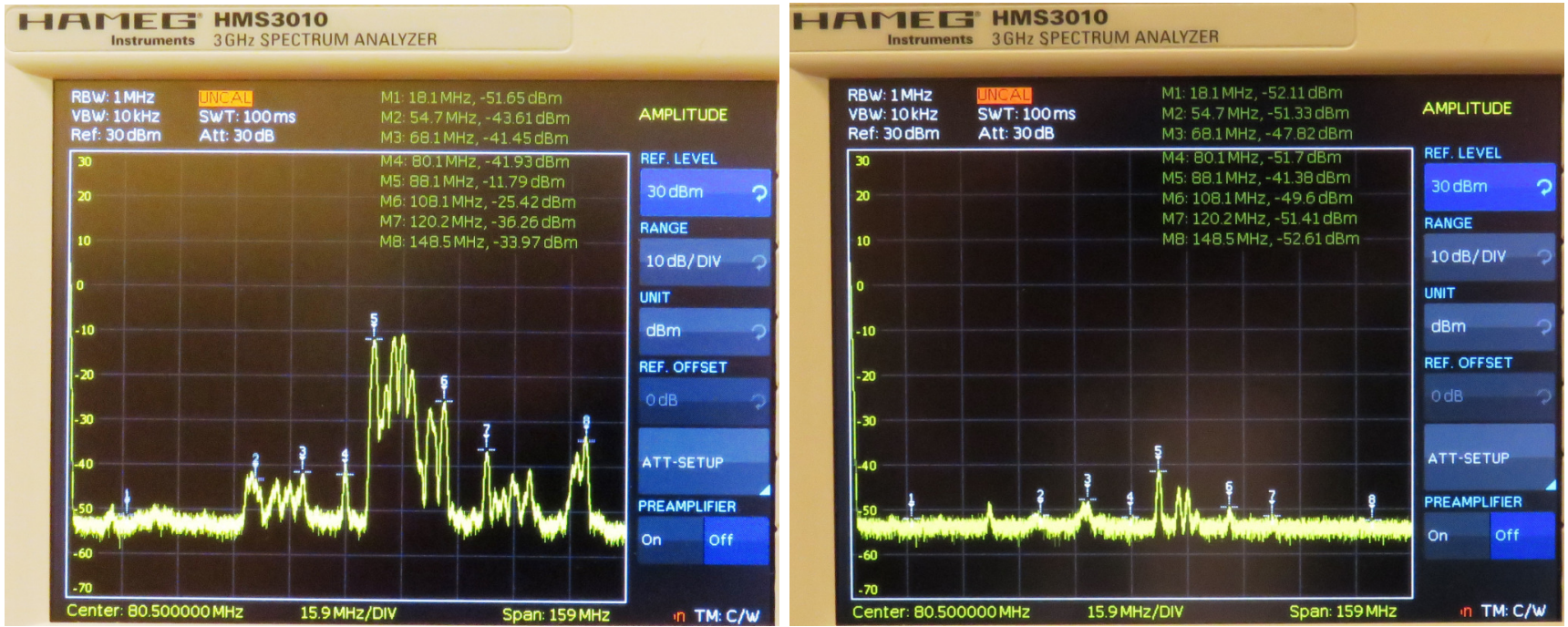

Fig. 14. Spectra of radio environment in Łódź direct from the antenna (left) and after low-pass Chebyshev analog filter (right)
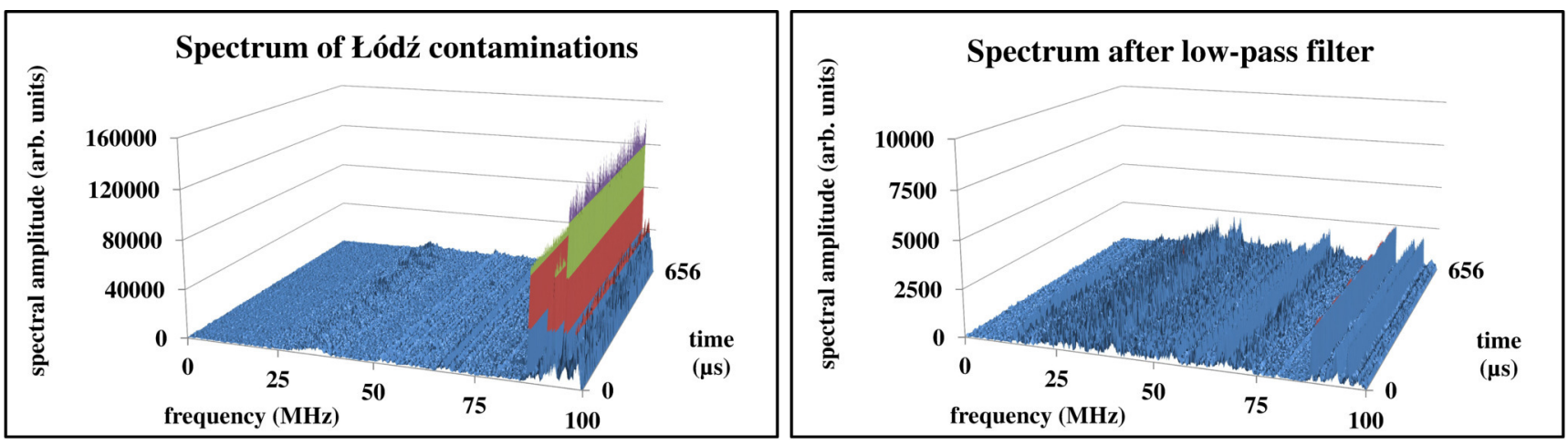

Fig. 15. Radio spectrum without (left) and with the filter (right) in the frequency range of 0-100 MHz. The sampling frequency equals $200 \mathrm{MHz}$.

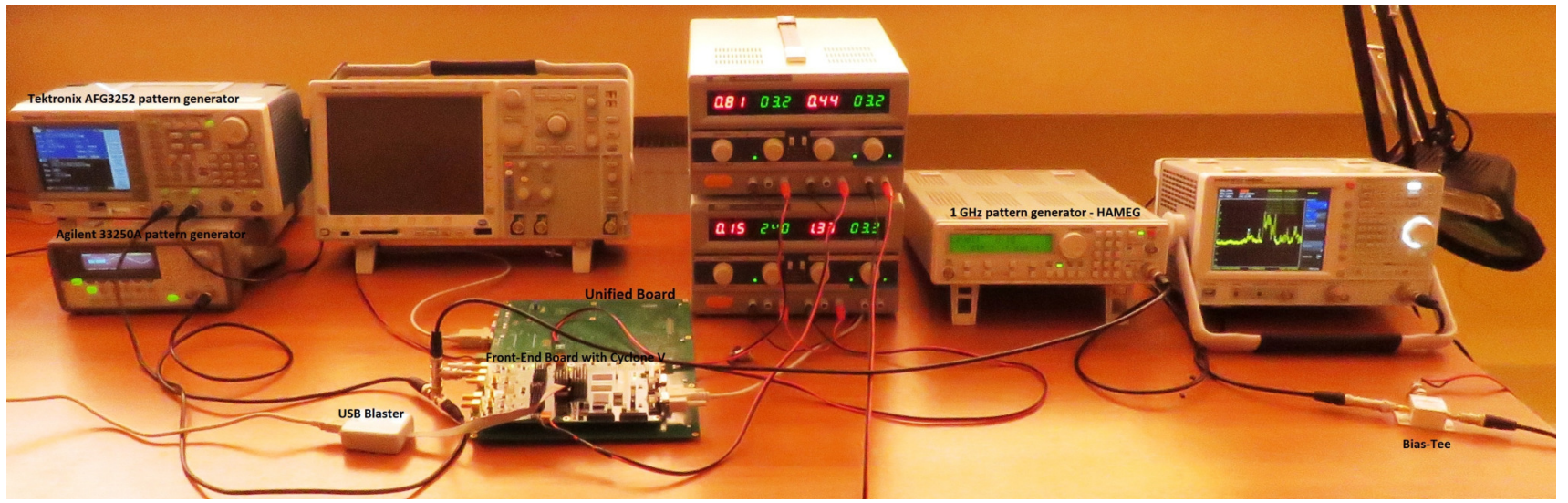

Fig. 16. Mesurement setup. Agilent 33250A, Tektronix AFG3252 and HAMEG pattern generators drive (as Wired-OR) the Front-End Board (FEB) with Cyclone ${ }^{\circledR}$ V FPGA 5CEFA9F32I7 as daughter board of the Unified Board (with micro-controller for a management of the Pierre Auger surface detector). The FEB is a prototype board for the surface detector but equipped with two radio channels with $200 \mathrm{MHz}$ sampling allowing a DAQ for AERA. The radio channel is driven either from from the pattern generators or from the AERA antenna via Bias-Tee.

AERA frequency characteristics due to the analog band-pass filter with 30-80 MHz cut-off. Fig. 17C shows that these peaks give a significant contribution to a total power. A task of Ex-NOR filtering is an elimination of pulses with spurious 

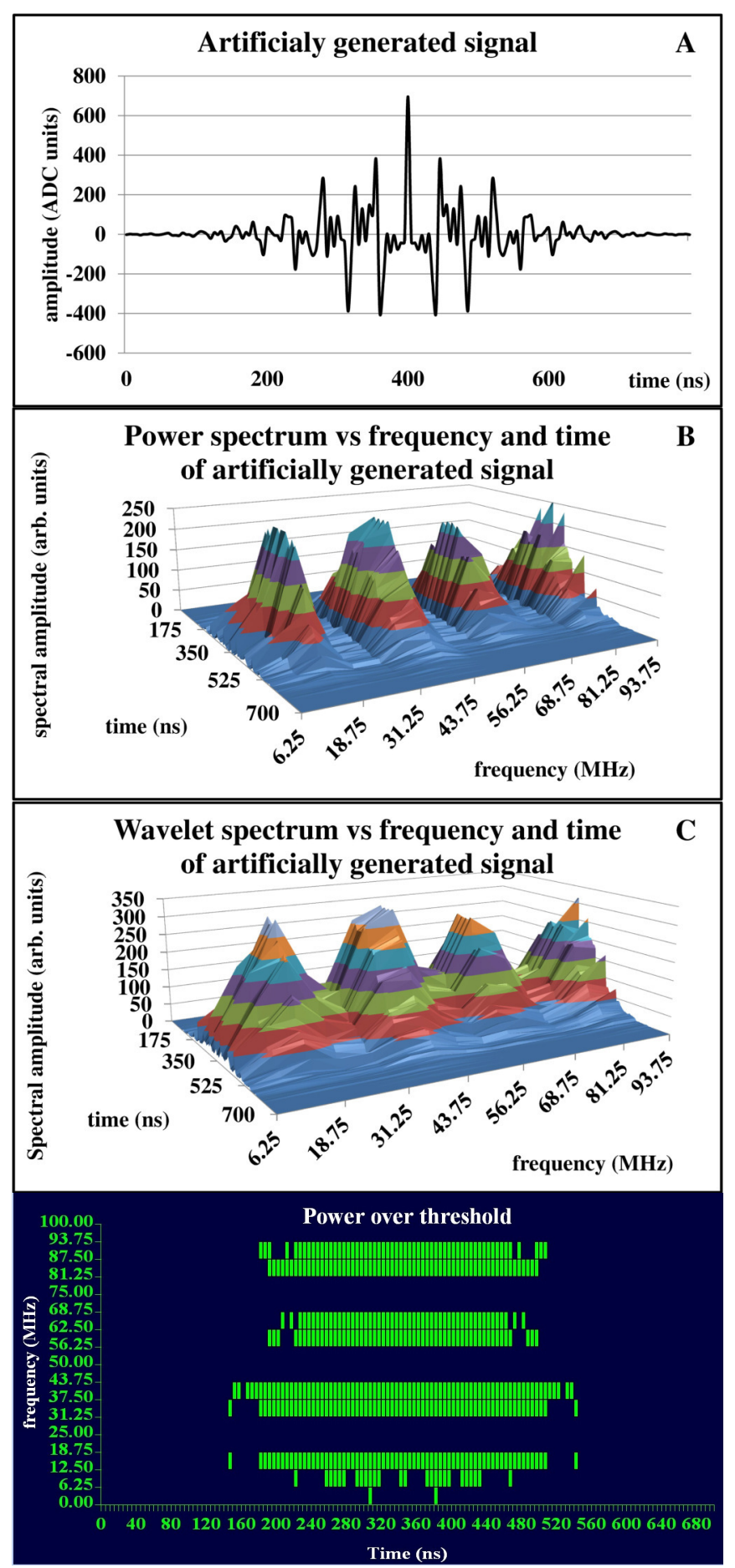

Fig. 17. Artificially generated pulse (A), its pure time/frequency spectrum without the wavelet scaling (B), the wavelet taking into account $(\mathrm{C})$ and power over threshold. Measured in the FPGA internal peaks $(B, C)$ and active regions (green regions in bottom graph) correspond to correct frequency range. Peripheral peaks correspond to spurious frequency leafs, which should not appear in real radio signals coming from cosmic rays (red regions). Amplitudes of peaks in graph B are reduced in comparison to Fig. 9. Taking into account wavelet coefficients to the power spectrum according to (4) peaks corresponding to the signals are soften with higher amplitude.

C
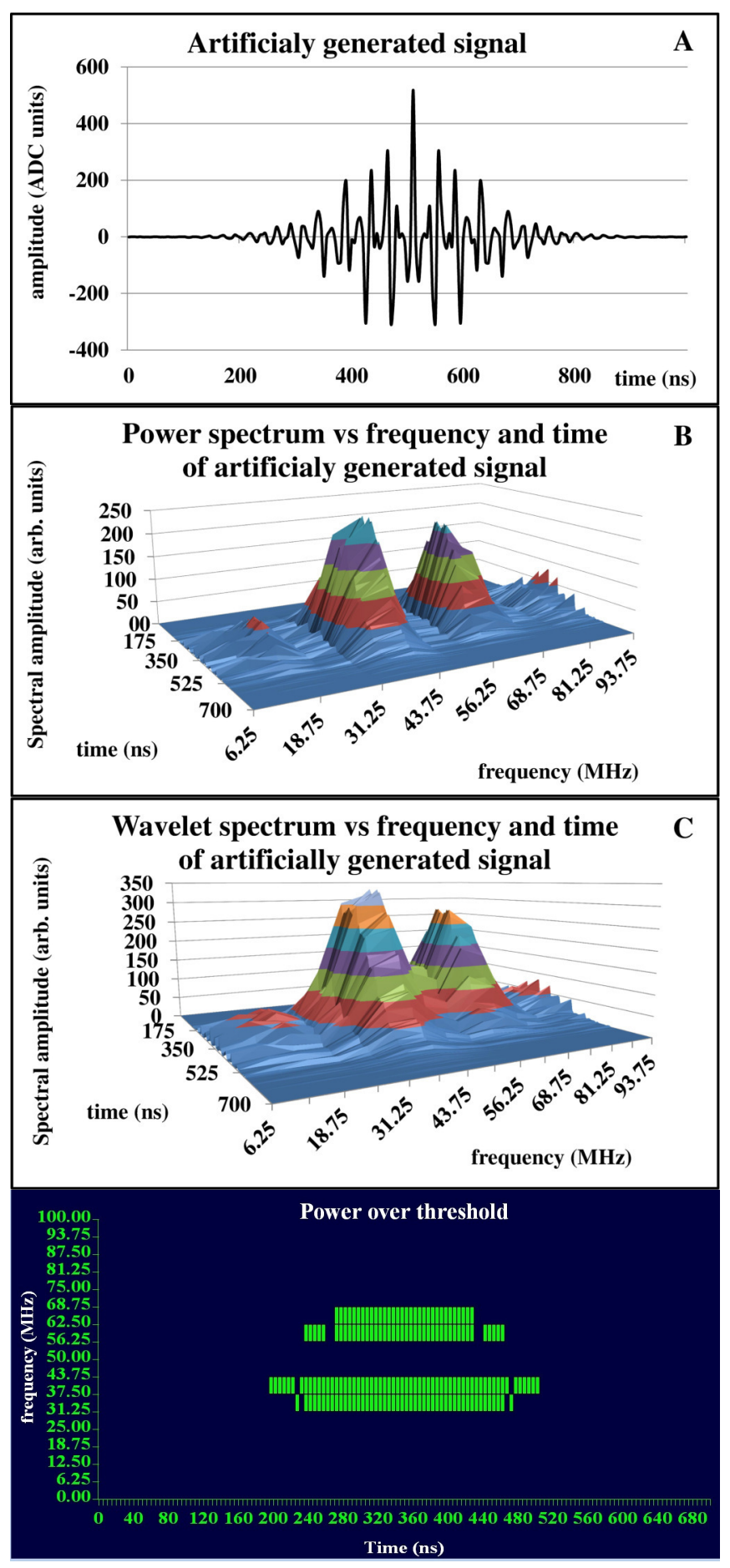

Fig. 18. Artificially generated pulse (A) with two peaks only. Description of the graph the same as for Fig. 17. Although the amplitudes $A_{0}$ and $A_{3}$ were reduced only twice (Fig. 9 - right graph), a selection of most significant bits by the NIOS almost eliminates peripheral contribution (graphs B and C). Only the green region appears in the bottom graph. The graph B shows the pure Fourier spectrum, the graph $\mathrm{C}$ with wavelet modification. The contribution of wavelets to the pure Fourier spectrum makes graphs soften, but peaks are amplified. 


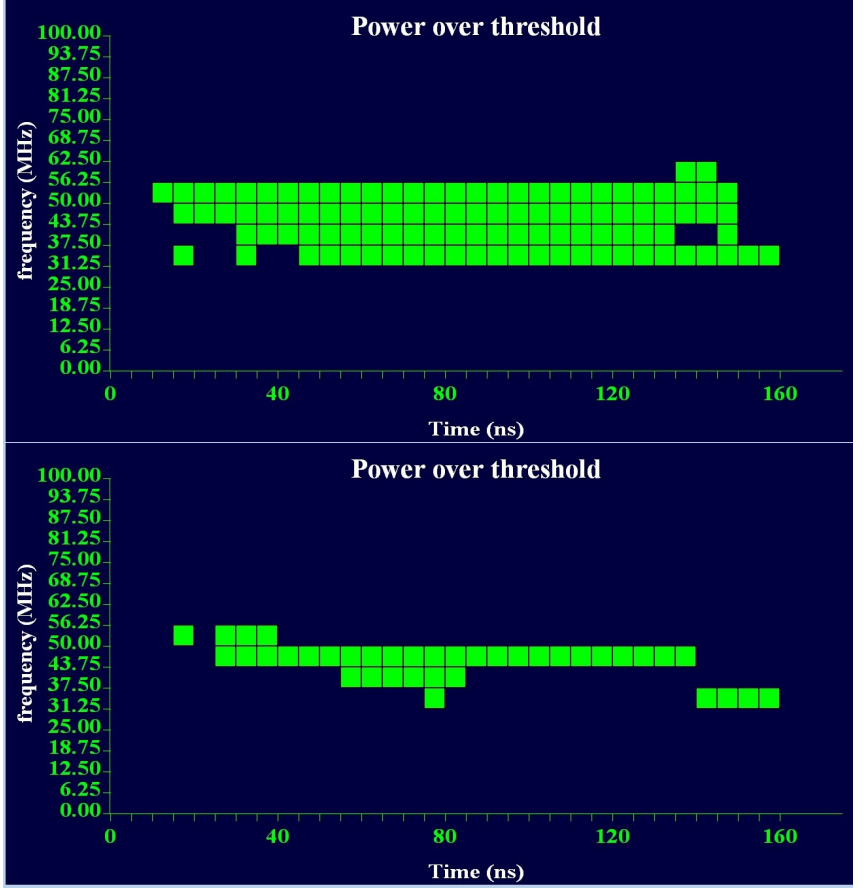

Fig. 19. Graphs presenting the power over threshold after the wavelet trigger Patterns from radio station LS035EW (upper) and LS005EW (lower) stored in the $256 \mathrm{kS}$ Agilent ROM are sent to the air via quarter-length dipole and received by the antenna.

frequency characteristics like in Fig. 17C.

Peripheral contributions are marked by the Ex-NOR filter as negative and they are subtracted from a positive contribution of internal peaks. Spurious regions are denoted in Fig. 17C by the red color. Because a contribution of peripheral (spurious) peaks is on the same level as internal one, the total power is negligible and this event is classified to be rejected.

However, if amplitudes $A_{0}=850$ and $A_{3}=900$ (in ADC units), respectively, the contribution of peripheral "bells" are much less and the peaks are below the threshold. Although there is some spurious contribution from peripherals, a final power is positive and such an event will be classified as potentially recognized cosmic-ray radio signal (Fig. 18).

\section{PRELIMINARY LABORATORY MEASUREMENTS}

Burst pulses from Tektronix AFG3252C arbitrary pattern generator have driven the radio channel in the prototype Front End Board (FEB) developed for the upgraded surface detector in the Pierre Auger Observatory [15][19]. The FEB is equipped with the biggest FPGA 5CEFA9F31I7 from the Cyclone ${ }^{\circledR} \mathrm{V}$ E family.

Signals from various radio stations may have significantly different amplitudes (compare Fig. 1A and 3A). The trigger algorithm has to take into account a wide amplitude range. $\left\|W_{n}\right\|$ modules outputs are put on a multiplexer dynamically selecting an optimal dynamic range. The multiplexer is controlled by the NIOS ${ }^{\circledR}$ processor. The $\bar{X}_{R e, I m}$ are 21-bit (seven pipeline stages for 32-point FFT) for 14-bit input ADC data $x_{n}$. To keep a maximal accuracy, intermediate pipeline results are not cropped. However, the product (5) for very wide inputs would be too wide and too sophisticated for an analysis. $\bar{X}_{R e, I m}$ are cropped to 16-bit range only (5 LSBs are neglected). The obtained $\left\|W_{n}\right\|$ is 32 bits. For an analysis, 16-bit window is selected by the NIOS ${ }^{\circledR}$ processor.

Fig. 3B shows a non-optimal selection (sel $=4$ as for LS005). Power peaks descended from noise are well visible. However, if an analysis window is shifted 2 bits higher, a frequency/time spectrum shows a pure peak descended from a real radio signal. The NIOS ${ }^{\circledR}$ processor periodically analyses a level of ADC noise and signal amplitudes and adjusts the multiplexer address for an optimal dynamic range (not to crop a signal and not to leave unused bits).

\section{Measurements with the AERA antenna}

The wavelet trigger was tested in the Łódź laboratory. Preamplifier as analog ASIC provided by SUBATECH was supplied from the antenna cable, via Bias-Tee ZFBT-282-1.5A from Mini-Circuit (Fig. 12). The Łódź radio environment is significantly contaminated by the high-power FM transmitters in the frequency range of $88-108 \mathrm{MHz}$ (Fig. 14 left). We used the Chebyshev low-pass filter to suppress significantly the frequency range above the Nyquist cut-off limit. Both HAMEG spectrum analyzer (Fig. 14 right) and the data analyzed by the Fron-End Board (Fig. 15 right) show that a contamination level coming from FM transmitter is now at the acceptable low level.

The pulses from Agilent arbitrary pattern generator (33250A) were being sent to the air by the quarter-length dipole and received by the butterfly antenna.

At first we tested the system by the artificial pulses with several peaks in the frequency spectrum (Fig. 17 and Fig. 18). The artificial pulses are much longer than the cosmic ray ones. Tests with real AERA pulses stored at first as patterns in the Agilent arbitrary pattern generator and next sent to the air by the quarter-length dipol and received by the butterfly antenna gave exactly the same distributions (Fig. 1B and Fig. 3C) as by direct driving from the FPGA ROM.

\section{CONCLUSION}

Laboratory tests show that the wavelet engine with the NIOS $^{\circledR}$ processor and set of registers, comparators and adders still provides a sufficient registered performance higher than $200 \mathrm{MHz}$ for Cyclone ${ }^{\circledR}$ IV E (EP4CE75F29C6) and Cyclone ${ }^{\circledR}$ V E (5CEFA9F31I7) FPGAs. Trigger operates according to our expectations.

We realize that the wavelet trigger utilizes a significant percent of FPGAs resources. Nevertheless, it is worth testing the algorithm in real radio stations in Argentinean pampas [20] using the Front-End Boards equipped with the standard AERA radio FEBs with EP4CE75F29C6 as a potential improvement of the AERA trigger for the next generation electronics. 


\section{ACKNOWLEDGMENT}

This work is supported by the National Science Centre (Poland) under NCN Grant HARMONIA-4 No. 2013/08/M/ST9/00322 and NCN Grant HARMONIA-8 No. 2016/22/M/ST9/00198

The author would like to thank the Aachen University for providing the AERA antenna and SUBATECH for the lownoise amplifier.

\section{REFERENCES}

[1] Pierre Auger Collaboration, A. Aab et al., The Pierre Auger Cosmic Ray Observatory, Nucl. Instrum. Meth. A 798 (2015) 172, DOI: 10.1016/j.nima.2015.06.058

[2] H.R. Allan, Radio Emission From Extensive Air Showers, Progress in Elementary Particle and Cosmic Ray Physics, vol. 10 (1971) 171.

[3] H. Falcke, P.W. Gorham, Detecting radio emission from cosmic ray air showers and neutrinos with a digital radio telescope, Astropart. Phys. 19 (2003) 477, DOI: 10.1016/S0927-6505(02)00245-1

[4] Pierre Auger Collaboration, A. Aab et al., Muons in air showers at the Pierre Auger Observatory: Measurement of atmospheric production depth, Phys. Rev. D90 Issue 1 (2014) 012012, DOI: 10.1103/PhysRevD.90.012012

[5] Pierre Auger Collaboration, S. Acounis et al., Results of a self-triggered prototype system at the Pierre Auger Observatory for radio-detection of air showers induced by cosmic rays, JINST 7 (2012) P11023, DOI: 10.1088/1748-0221/7/11/P11023

[6] Pierre Auger Collaboration, A. Aab et al., Probing the radio emission from air showers with polarization measurements, Phys. Rev. D89 (2014) 052002, DOI: 10.1103/PhysRevD.89.052002

[7] S. Fliescher for the Pierre Auger Collaboration, Radio detection of cosmic ray induced air showers at the Pierre Auger Observatory, Nucl. Instrum. Meth. A662 (2012) S124, DOI: 10.1016/j.nima.2010.11.045

[8] R. Dallier for the Pierre Auger Collaboration, Measuring cosmic ray radio signals at the Pierre Auger Observatory, Nucl. Instrum. Meth. A630 (2011) 218, DOI: 10.1016/j.nima.2010.06.069
[9] T. Huege for the Pierre Auger Collaboration, Radio detection of cosmic rays in the Pierre Auger Observatory, Nucl. Instrum. Meth. A617 (2010) 484, DOI: 10.1016/j.nima.2009.10.012

[10] Pierre Auger Collaboration, J. Abraham et al., The fluorescence detector of the Pierre Auger Observatory, Nucl. Instrum. Meth. A620 (2010) 227, DOI: 10.1016/j.nima.2010.04.023

[11] Pierre Auger Collaboration, P. Abreu et al., Antennas for the Detection of Radio Emission Pulses from Cosmic-Ray induced Air Showers at the Pierre Auger Observatory, JINST 7 (2012) P11011, DOI: 10.1088/17480221/7/10/P10011

[12] I. Daubechies, The wavelet transform time-frequency localization and signal analysis, IEEE Trans. Inform. Theory 36 (1990) 961.

[13] Z. Szadkowski, An Optimization of the FPGA Based Wavelet Trigger in Radio Detection of Cosmic Rays, IEEE Trans. on Nucl. Science 62 Issue 3 (2015) 993, DOI: 10.1109/TNS.2015.2422713

[14] C. Torrence, G.P. Compo, A Practical Guide to Wavelet Analysis, Bulletin of the American Meteorological Society 79 (1998) 61.

[15] Z. Szadkowski, Front-End Board with Cyclone $V$ as a Test HighResolution Platform for the Auger Beyond 2015 Front End Electronics, IEEE Trans. on Nucl. Science 62 Issue 3 (2015) 985, DOI: 10.1109/TNS.2015.2426059

[16] Z. Szadkowski, A. Szadkowska, FPGA Based Wavelet Trigger in Radio Detection of Cosmic Rays, Braz. J Phys 44 (2014) 805, DOI: 10.1007/s13538-014-0243-5

[17] Z. Szadkowski, 16-point discrete Fourier transform based on the Radix-2 FFT algorithm implemented into cyclone FPGA as the UHECR trigger for horizontal air showers in the Pierre Auger Observatory, Nucl. Instrum. Meth. A 560, Issue 2 (2006) 309, DOI: 10.1016/j.nima.2006.01.045

[18] Pierre Auger Collaboration,, P. Abreu et al., Antennas for the detection of radio emission pulses from cosmic-ray induced air showers at the Pierre Auger Observatory, JINST 7 (2012) P10011.

[19] Z. Szadkowski, First results of High-Resolution Front End Electronics for Water Cherenkov Air Shower Detectors equipped with Cyclone V FPGA, IEEE Trans. on Nucl. Science 63 Issue 3 (2016) 1446, DOI: 10.1109/TNS.2016.2567320

[20] Pierre Auger Collaboration, J. Abraham et al., Atmospheric effects on extensive air showers observed with the surface detector of the Pierre Auger Observatory, Astropart. Phys. 32 (2009) 88, DOI: 10.1016/j.astropartphys.2009.06.004 